\title{
WestVirginiaUniversity
}

THE RESEARCH REPOSITORY @ WVU

Graduate Theses, Dissertations, and Problem Reports

2012

\section{VCAST: A Distance-Sensitive Scalable Information Dissemination Protocol}

Raja Abhinay Moparthi

West Virginia University

Follow this and additional works at: https://researchrepository.wvu.edu/etd

\section{Recommended Citation}

Moparthi, Raja Abhinay, "VCAST: A Distance-Sensitive Scalable Information Dissemination Protocol" (2012). Graduate Theses, Dissertations, and Problem Reports. 4898.

https://researchrepository.wvu.edu/etd/4898

This Thesis is protected by copyright and/or related rights. It has been brought to you by the The Research Repository @ WVU with permission from the rights-holder(s). You are free to use this Thesis in any way that is permitted by the copyright and related rights legislation that applies to your use. For other uses you must obtain permission from the rights-holder(s) directly, unless additional rights are indicated by a Creative Commons license in the record and/ or on the work itself. This Thesis has been accepted for inclusion in WVU Graduate Theses, Dissertations, and Problem Reports collection by an authorized administrator of The Research Repository @ WVU. For more information, please contact researchrepository@mail.wvu.edu. 


\title{
VCAST: A Distance-Sensitive Scalable Information Dissemination Protocol
}

\author{
by
}

Raja Abhinay Moparthi

Thesis submitted to the

Benjamin M. Statler College of Engineering and Mineral Resources at West Virginia University

in partial fulfillment of the requirements

for the degree of

\author{
Master of Science \\ in \\ Electrical Engineering \\ Vinod K. Kulathumani, Ph.D., Chair \\ Yenumula V. Reddy, Ph.D. \\ Arun A. Ross, Ph.D.
}

Lane Department of Computer Science and Electrical Engineering
Morgantown, West Virginia
2012

Keywords: Vehicular Networks, VCAST, Simulation

Copyright 2012 Raja Abhinay Moparthi 


\begin{abstract}
VCAST: A Distance-Sensitive Scalable Information Dissemination Protocol
\end{abstract}

by

\author{
Raja Abhinay Moparthi \\ Master of Science in Electrical Engineering \\ West Virginia University \\ Vinod K. Kulathumani, Ph.D., Chair
}

The future of intelligent transportation systems lies in dealing with safety and navigation of vehicles. Building a technology that provides the real-time information about the state of other vehicles like its location, speed and direction would help in developing a system that ensures safety along with navigation. However, the network limitations pose difficulty in obtaining the state information over multiple-hops, because of the bandwidth limitations and congestion in the shared wireless channel. Overcoming this challenge would yield an intelligent transportation system which gives information regarding the collisions, lane changes and merges, emergency vehicle approaching alerts, stopped vehicle alerts, etc., over larger distances. In my thesis I developed an algorithm VCAST that addresses this challenge by considering the grounds that the response time needed by vehicles at farther distances is more than that of at the smaller distances. This fact exploits the notion of distance sensitivity in information propagation, in which information is forwarded at a rate that decreases linearly with distance from source. The algorithm outputs traffic information with staleness, a measure of error in traffic information, bounded by $O\left(d_{h}^{2}\right)$, where $d_{h}$ is the single communication hop range. Also the communication rate per vehicle per unit time depends on the area of consideration but not on the density or number of vehicles in the region, which can be further reduced by considering the aggregated information over smaller regions. Thus, this technique would be able to supply timely information over large distances without compromising on data rates at smaller distances. Also, VCAST doesn't need any special hardware or changes to the vehicular transmission standards. We evaluate the performance of VCAST by simulating a 4-lane highway of $5 \mathrm{Kms}$ occupied by 800 vehicles, wherein we vary the densities with and without fading apart from aggregated information propagation using the IEEE 802.11p communication model on NS-3. 


\section{Acknowledgements}

This thesis would not have been possible without the guidance from several individuals who directly or indirectly supported me throughout this study.

I would like to express my deepest appreciation to my advisor, Dr. Vinod Kulathumani for granting me this opportunity. His supervision and guidance from the very early stage of my research is worth mentioning. His expertise, valuable suggestions and insights are truly commendable.

I am grateful to Dr. Yenumula V. Reddy and Dr. Arun A. Ross for their unconditional support and time throughout the program.

Many thanks would go to my research group members Sriram Sankar, Rahul Kavi, Rudhir Upretee and Goutham Ranganath for their encouragement and support. They played their part in making the work environment fun and able place.

Finally, a special thanks to my parents, family members and friends who always stood by my side at all times. I am always indebted to them. 


\section{Contents}

Acknowledgements

List of Figures $\quad$ v

$\begin{array}{ll}\text { Introduction } & 1\end{array}$

1.1. Motivation 1

1.2. Impact $\quad 4$

1.3. Thesis Outline 5

$\begin{array}{lc}\text { Related Work } & 6\end{array}$

2.1. Optimization of Communication Ranges 6

2.2. Collision Avoidance $\quad 7$

2.3. Redundancy Avoidance $\quad 7$

2.4. Distance Sensitivity 9

$\begin{array}{ll}\text { VCAST Algorithm } & 10\end{array}$

3.1. System Description and Objective 10

3.2. Algorithm: 11

3.3. Analysis 14

$\begin{array}{lr}\text { Network Simulator-3 (NS3) } & 18\end{array}$

4.1. Basic elements of Network Simulator-3 18

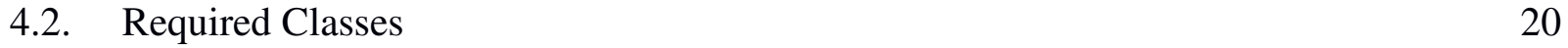

4.3. Modules for VCAST Simulation $\quad 21$

$\begin{array}{ll}\text { Results \& Observations } & 26\end{array}$

5.1. Fixed inter-vehicular separation 27

5.2. Impact of time varying inter-vehicular separations 29

5.3. Impact of fading in conjunction with variable speed 30

$\begin{array}{ll}\text { 5.4. Impact of density } & 31\end{array}$

5.5. Analysis of communication cost $\quad 32$

5.6. Impact of aggregation 33

$\begin{array}{ll}\text { Conclusions } & 36\end{array}$

$\begin{array}{ll}\text { References } & 38\end{array}$ 


\section{List of Figures}

Fig 1.1: Utilization of multi-hop vehicular information for safety applications. The arrows indicate multi-hop information flow towards car C. Knowledge of states of $\mathrm{A}$ and $\mathrm{D}$ will guarantee safe lane change to left and right respectively. Knowledge of congestion (shown by a dense cluster of vehicles) will allow vehicle $\mathrm{C}$ to progressively decrease its speed ensuring a safe and timely reaction.

Fig 5.1(a): Maximum staleness vs. pair-wise vehicular distance: $\mathrm{p}=5 \mathrm{~Hz}$, one hop communication range of $200 \mathrm{~m}, 300 \mathrm{~m}, 400 \mathrm{~m}$ and $500 \mathrm{~m}$.

Fig 5.1(b): Maximum staleness vs. pair-wise vehicular distance: $\mathrm{p}=20 \mathrm{~Hz}$, one hop communication range of $200 \mathrm{~m}, 300 \mathrm{~m}, 400 \mathrm{~m}$ and $500 \mathrm{~m}$.

Fig 5.2: Maximum staleness vs. pair-wise vehicular distance: $\mathrm{p}=20 \mathrm{~Hz}$ at smaller distances (Zoomed-in version of Fig. 5.1(b)) 28

Fig 5.3(a): Maximum staleness vs. pair-wise vehicular distance: $\mathrm{rh}=300 \mathrm{~m}$

Fig 5.3(a): Maximum staleness vs. pair-wise vehicular distance: $\mathrm{rh}=500 \mathrm{~m}$

Fig. 5.4: Impact of time-varying vehicular separations 30

Fig. 5.5: Impact of channel fading 30

Fig. 5.6: $\quad$ Impact of network density 31

Fig 5.7: Analysis of communication cost. Denstiy $=25 \mathrm{~m}$ separation. 32

Fig 5.8: Distribution of communication load across vehicles in the network; $\mathrm{p}=5 \mathrm{~Hz}$, $\mathrm{rh}=500 \mathrm{~m}$

Fig 5.9: Analysis of communication cost $\quad 34$

Fig. 5.10: $\quad$ Impact of information aggregation. 34 


\section{Chapter 1}

\section{Introduction}

\subsection{Motivation}

Most latest technological advancements in the field of automobiles have made commuting safer and efficient [1]. An example of this is the in-vehicle communication system which is controlled by electronic devices using Controller Area Networks (CAN's). This includes maintenance alerts, security alerts, cruise control systems, etc. These systems collect data from numerous sensors placed at various locations on the vehicle and this data, after processing, is presented to driver via on-board display simultaneously actuating the required mechanisms within the vehicle and thereby enabling safety and efficiency. Another form of communication is the system which connects the vehicle with the satellites, like the ON-STAR system employed by General Motors. This is useful in performing actions like hands-free calling, turn-by-turn navigation, remote diagnostics, automatic crash response and stolen vehicle tracking.

Apart from these present systems, there is a necessity for a system which enables communication between vehicles. This enhances vehicle-to-vehicle safety better than any other system in place today. In either cases of completely autonomous or semi-autonomous systems, vehicles move with speeds at which it becomes difficult to control with precision and timing, which may not be possible with low response times. The in-vehicle communication system only monitors the area around the car within a very small range whereas the vehicle-to-vehicle communication system provides us with knowledge of traffic patterns, road conditions beforehand over farther distances there by enabling safety and intelligent transportation system. Examples of safety application in transportation scenarios include collision warnings, guidance on lane change and lane merge, stopped vehicle and emergency vehicle alerts, etc. Examples of 
intelligent navigation applications include dynamic travel-time computations and re-routing based on real-time traffic information.

As per the statistics of National Highway Traffic Safety Administration (NHTSA), it was said that the Vehicle to Vehicle systems potentially address about 4.4 million crashes annually in USA [1]. If this is taken to be the primary counter measure, it is said that $74 \%$ of the overall crashes can be dealt with a Vehicle-to-Vehicle Communication system involving all of the vehicle types.

Vehicular Networks plays a major role in the Automated Vehicular Systems, where there is no need of human effort in driving the vehicle, rather the computers that were built into the vehicles take the information from different sensors fixed all over the vehicle's body and drive the vehicle on the traffic lanes. Along with the data from the sensors, if the computer receives the information from its neighboring vehicles and makes decisions using this information, it would result in better safety, accuracy and smoothness in operation. Google's self-driving car is an example of completely autonomous vehicle. The safety and intelligent transportation applications help the systems on the autonomous vehicle to adjust the speed according to the road conditions and thereby having a smooth operation.

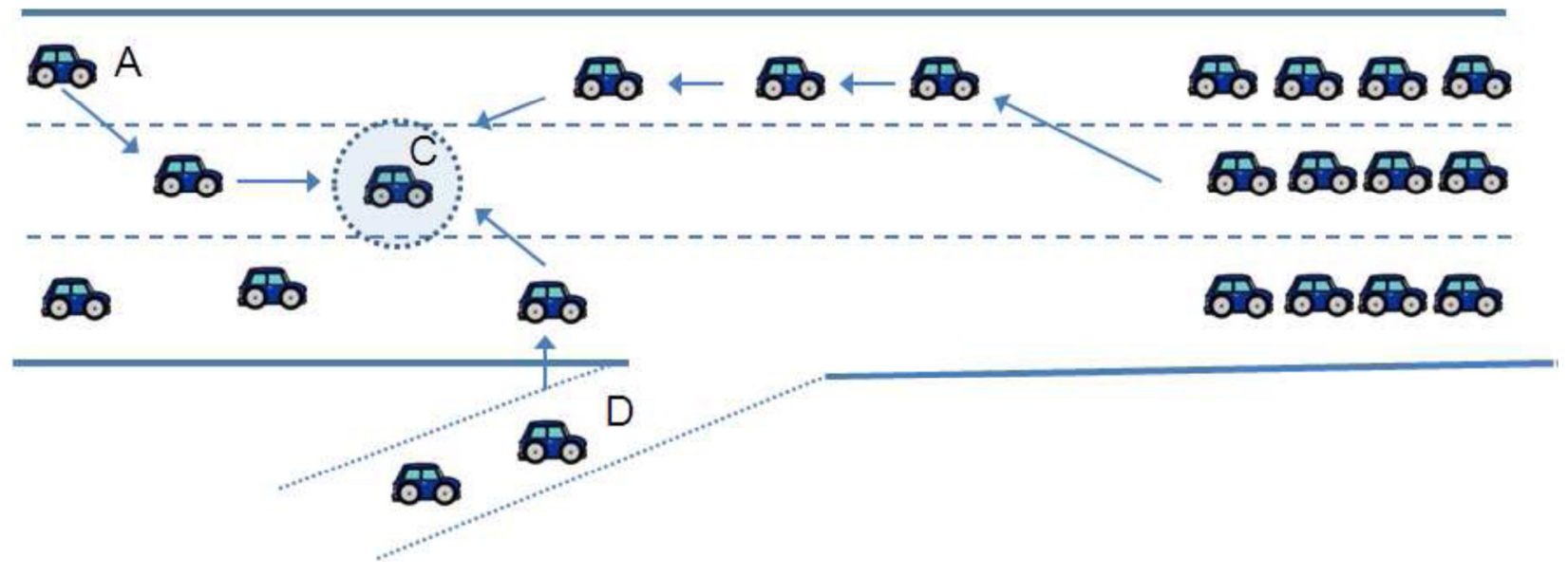

Fig 1.1: Utilization of multi-hop vehicular information for safety applications. The arrows indicate multi-hop information flow towards car $C$. Knowledge of states of $A$ and $D$ will guarantee safe lane change to left and right respectively. Knowledge of congestion (shown by a dense cluster of vehicles) will allow vehicle $\mathrm{C}$ to progressively decrease its speed ensuring a safe and timely reaction. 
Fig. 1.1 gives the idea of how the VCAST system helps the vehicle operator in different situations through the multi-hop information propagation. The information passed by car D to car $\mathrm{C}$ helps it to know the lane merging situation of car D and hence enables to take decision depending on the speeds and point of merging. At the same time car $\mathrm{C}$ gets the information from car A over multiple-hops regarding the emergency vehicle. The congestion information from the cars at the congested region is transferred over multi-hop to car $\mathrm{C}$ such that there is enough of the response time in lowering the speed of vehicle there by having a smooth drive instead of jerky stops.

The building block of all these applications is the onboard vehicular traffic mapping system, which portrays information about current position of other vehicles in its vicinity and provides guidance about accidents, approaching emergency vehicles and traffic congestion over an extended neighborhood. To build a system with such functionalities, we assume that each vehicle is equipped with a GPS (Global Positioning System) onboard, so that it estimates the location to an accuracy of 1-2 meters. This information is advertised in its one hop communication neighborhood [6], which is then forwarded by the neighboring vehicles over multi-hop. The simple case of forwarding all the information would look good in case of idle scenario, but practically introduces many limitations on the wireless communication and is a challenging task to estimate the traffic maps over a large area using multi-hop wireless communication. Also, the technique of forwarding each vehicle's information over multiple hops at a constant rate may not scale well as the communication overhead per each node increases with increase in density and size of the tracking region [2][3]. Hence there need to be an efficient forwarding algorithm designed in order to ensure that the system is scalable with vehicle density and the area of interest. On the other hand, there happens to be a tradeoff between the rate of communication and the broadcast range. As an observation, it can be inferred that higher broadcast rate and range would yield greater tracking accuracy, but in practice, the tracking accuracy goes down after certain limit because of the wireless channel contentions. Existing broadcast techniques approach this issue by balancing the transmission rate and communication range over single hop for maximum reliability [12]. Such an approach would result in the information availability to the neighboring vehicles only when the inter-vehicular distance is very small which may not be enough to avert a collision [3]. Moreover compromising with the transmission rate would decrease the achievable information supply rate even at smaller 
communication distances thus making the nearby vehicles get less accurate information. Hence, there is a need of a multi-hop broadcasting technique where the information regarding the farther nodes is updated in a timely fashion without compromising with data rates at smaller distances.

We introduce VCAST as a solution to this problem which scales well with the changes in vehicular densities and tracking region by delivering the required data rates at smaller distances with required information availability at farther distances. The designed algorithm adopts a method of distance-sensitive propagation of individual vehicle's information; where the information about each vehicle is propagated at a rate that decreases linearly with distance from the source vehicle [4][5]. The fundamental reason for exploiting the distance-sensitivity in information forwarding is that the response times available for a vehicle to make a safe decision or for computing a new route is lesser for vehicles at smaller distances than that of the vehicles at longer distances [3].

\subsection{Impact}

Thus we derive that the information about the vehicles in near vicinity region is more frequently updated than the vehicles in farther vicinity region. Still this is said to be a sufficient condition for having vehicular safety and intelligent navigation. The advantages of such an technique are: (1) information regarding vehicle location is available even if the communication range is very low because of the higher density, which is the case seen in urban traffic situations, (2) the lane changing information is available even if the vehicles are outside a single communication region, (3) approaching emergency vehicles information will be available beforehand, (4) based on the received information the system can dynamically reroute if there are any road works, blocks, accidents and impending congestion. For all these applications the VCAST algorithm makes a compromise between the accuracy and distance ensuring that it is still sufficient for applications like vehicular safety and intelligent navigation. 


\subsection{Thesis Outline}

In chapter 2, prior to going into the details of the VCAST and its simulation we did some literature review explaining how the unicast, multicast and broadcast techniques fail to scale well for information dissemination. We studied different broadcasting algorithms and the effect of increase in density and area on their performance.

This is followed by the explanation of VCAST algorithm in chapter 3, where we adopted an intelligent approach in eliminating the effect of density and area in case of IVC (Inter Vehicular Communications), for information dissemination. We presented that in the form of theorems and explaining each of these theorems with logical and mathematical proofs.

Chapter 4 explains about the Network Simulator 3 that we used for simulating the VCAST algorithm and the basic entities of the network simulator. This is followed by the explanation of modules that play role in the simulation.

In Chapter 5 we discussed about the results that obtained from the simulation of VCAST and the conclusions made from the resulting graphs are documented. 


\section{Chapter 2}

\section{Related Work}

Much of the research have been going the recent times on the vehicular networks and many solutions have been developed for having an effective communication system between moving vehicles. A number of protocols were proposed in the field of vehicular ad-hoc networks, and particularly mobile ad-hoc network (MANET) has become a well-researched topic. All these protocols concentrate on aperiodic, low bandwidth data such as emergency vehicle information to either a single vehicle (unicast) or a group of vehicles in a geographic region (geocast) with low latency [7][8][9]. But we are interested in broadcasting information by each and every vehicle at higher transmission rates over larger regions.

\subsection{Optimization of Communication Ranges}

With the need for the wireless broadcasting in the vehicular ad-hoc networks for safety application, a lot of research attention has been received lately. The solutions proposed in these papers concentrated mainly on optimizing the communication range with greater reliability for less number of data collisions and successful receptions [12][13][14]. For this to happen, the communication range or the power of transmission is reduced with the increase in the density of the vehicles. Hence the volume of data per unit area in the network decreases which in turn leads to lesser number of collisions resulting in the growth of successful receptions. The problem with this type of piggyback method of power and transmission distance control methods is that it becomes difficult to stabilize for a system like vehicular networks, where mobility happens at a faster rate and the configuration of system changes at higher rate. Along with these factors, there is a higher staleness in the information availability for the nearby vehicles with higher communication ranges and a lower communication range prevents the information availability from beyond the range. 


\subsection{Collision Avoidance}

In the case of multi hop networks where the flooding of data takes place, the success rate for the information dissemination will be high only if the density of vehicles is low [15][17]. The proposed MHVB algorithm uses the flooding that depends on the distance between sender and receiver. In this technique the receiver calculates the distance of sender from itself using the information received. If the sender is greater than the threshold it doesn't forward the source information. Otherwise the receiver forwards it with delay depending upon the distance of sender. For farther distances, information is forwarded immediately, but for the adjacent or nearer distances, source information it forwards with some delay. By doing so, the data collisions in the network decreases as all the nodes which receive the information doesn't try to forward the information as soon as they get the data. Even with this approach, as vehicular density increases the success rate of reception decreases. On the other hand, in VCAST, the multi hop message forwarding algorithm would enable a vehicle to know about its nearby vehicles which may or may not be in its communication range. All this happens with lower communication rate at each node by which it reduces the data traffic in the network. Also this algorithm enables forwarding the aggregated information of a set of vehicle in a cell by which the communication rate can be further reduced.

\subsection{Redundancy Avoidance}

The paper 'Comparison of Broadcasting Techniques' compares different data dissemination mechanisms in MANETS (Mobile Adhoc Networks) that reduce the number of redundant transmissions and channel traffic [18]. The simple flooding technique where nodes just forward the message that it received from neighbors is explained only with respect to low node density and/or high mobility. The probabilistic scheme model is similar to the simple flooding except that nodes only rebroadcast with predetermined probability [16]. This shares similar transmission coverage in dense networks. Making rebroadcast on a random basis would save node and network resources without harming delivery effectiveness. Adverse of this is seen in the sparse networks where there will be a much less shared coverage leading to non-reception of all broadcast packets even at shorter distances. This protocol would not yield a good efficiency in the case of vehicular networks as the nearest vehicles information may not be received at all times. The Counter-Based Scheme has the advantage of adapting to both the 
highly dense area of network as well as the sparse areas of the network. Every node maintains a counter and starts a timer that fires randomly between 0 to $\mathrm{T}_{\max }$ seconds. Meanwhile the node increments the counter corresponding to each received packet of nodes information. If the counter value is below some threshold at the end of timer, then the node rebroadcasts, otherwise neglects it. This technique does concentrate only on the extra redundant traffic in the channel at higher densities but doesn't scale well on normalized data traffic with the higher density in network.

The other mode of data broadcasts involves Area Based Methods and Neighbor Knowledge Methods [11]. When considering the general simple broadcast mechanism, if the receiving node that is located just few meters away from the sender tries to rebroadcast the received information, the additional area covered by this retransmission is quite low. On the other extreme if a node is located at the border of transmission distance then a rebroadcast would reach significant additional area and also reduces the traffic per unit area in a network. The area based models are implemented in two different schemes namely Distance-Based scheme and Location-Based scheme. A node using Distance-Based scheme compares the distance between itself and each neighbor node that has previously rebroadcast a given packet for making a decision on the rebroadcast by itself. On the other side the Location-Based scheme uses the Global Positioning System (GPS) for calculating the effective additional area of coverage after rebroadcast and makes the decision on rebroadcasting.

Apart from the Area based and Probability based methods there are the broadcast techniques with Neighbor Knowledge. Flooding with Self Pruning is the simplest of this which uses "Hello" packets for the exchange of knowledge regarding 1-hop neighbors. Every node that broadcast a packet includes the list of known neighbors in the header. Thus the receiver of the packet checks for any additional node that would reach on rebroadcast and make appropriate decision. But the problem with this type of protocol is that the network overhead increases with increase in density as the number of neighboring nodes list would grow high. Moving to the Scalable Broadcast Algorithm, the 2-hop neighbor knowledge is taken in to consideration. The Dominant Pruning technique do use the same 2-hop pruning technique expect that the header of each packet is modified by adding the address of each sender. By doing so the receiver checks for its address in the header and makes a decision by using Greedy Set Cover algorithm where it 
recursively chooses 1-hop neighbors which cover the most 2-hop neighbors and recalculates the cover set until all 2-hop neighbors are covered. The Multipoint Relaying mechanism is similar to Dominant Pruning technique except that instead of rebroadcasting by all the one hop neighbors only the selected one hop neighbors are selected based on effective coverage of nodes called Multipoint Relays (MPRs).

All these proposed protocols for broadcasting speak regarding the redundancy avoidance in the channel but doesn't concentrate on the information availability rate at different distances thus decreasing the information overhead over nodes. Also these proposed mechanisms don't scale well with increase in vehicle density and tracking region leading to the improper distribution of the information overhead over nodes. All these factors are answered with the idea of distance-sensitive broadcasting technique where the system scales well with higher broadcast rates and all - all information broadcast, that has not been addressed in any of the mobile ad-hoc and vehicular networks.

\subsection{Distance Sensitivity}

The distance sensitive approach has found applications in several other networking fields. For the efficient distributing routing information the distance sensitive mechanism is used for route aggregation in Internet. Fisheye routing uses this idea to propagate routing tables in mobile ad-hoc networks. The application of distance sensitivity is also used in wireless sensor network based querying and tracking applications to model communication latency and information quality with respect to distance [21]. Using the distance sensitivity in VCAST we show that it is a valuable tool for efficient and scalable propagation of state information even for networks of mobile nodes. 


\section{Chapter 3}

\section{VCAST Algorithm}

The VCAST algorithm that was developed has a distance sensitive forwarding mechanism. The information of the vehicle at a distance of ' $h$ ' hops will be forwarded only once in every ' $h$ ' transmission cycles. That is, the rate of forwarding decreases linearly with the distance of source from the forwarding vehicle. We use staleness in vehicular state information as a metric for information quality as it reflects how old the current information about a particular vehicle is. Implementing an algorithm like this would yield you the traffic information with a staleness bounded by $O\left(d_{h}{ }^{2}\right)$ where ' $d_{h}$ ' is the hop distance from the information source to the receiving vehicle. Also the average communication cost does not grow with the vehicular density in a region, and is only bounded by $O\left(R_{h} . p\right)$, where ' $R_{h}$ ' is the maximum number of communication hops in a region over which traffic information is to be propagated and ' $p$ ' is the broadcast rate.

The communication rate per node can be further decreased by forwarding only the aggregated information rather than individual vehicle details. This is done by considering predefined parts of the region (a couple of hundreds of meters, called a cell) and aggregating the individual information in that cell and forwarding that aggregated information. As a result, the communication rate further decreases by a factor $r_{c}$, where ' $r_{c}$ ' represents the radius of each cell.

\subsection{System Description and Objective}

We considered our system of vehicular network to have a large geographical area with a number of vehicles non-uniformly distributed over the entire region and having diversified traffic patterns. Each vehicle needs to maintain the information regarding every other vehicle located at a maximum distance of ' $R$ '. The maximum density at any time over this region is considered as ' $\rho$ '. The geographic region is divided into cells for having the aggregated traffic information. These cells may or may not be uniform but we assume it to be uniform for making things easier. Thus the area of each cell is a constant ' $A_{c}$ ', and the radius of each cell is ' $r_{c}$ '. In order to have a real scenario the entire geographic location may have zero traffic density i.e., no roads and have high density traffic patterns. The communication range of each of the vehicle is $r_{h} .\left(r_{h}<<\mathrm{R}\right)$. 
The primary objective of our system is to track the vehicles around it which are at a maximum distance of $\mathrm{R}$ by receiving information through the intermediate vehicles.

If ' $N$ ' denotes the total number of nodes in the tracking region of the vehicle then $N=\rho \pi R^{2}$. If ' $L$ ' is the number of cells in the region with radius ' $R$ ', i.e., the tracking region, then $L=\frac{\pi R^{2}}{A_{c}}$. Assume that a vehicle transmits its own information every ' $p$ ' times in second, which implies that the broadcasting frequency of the vehicle is ' $p$ ' $H z$. If $i$ and $j$ denote two vehicles departed apart by a geographical distance of $d(i, j)$ and are $d_{h}(i, j)$ hops away from one another then it can be given that $d_{h}(i, j)=\frac{d(i, j)}{r_{h}}$, where $r_{h}$ as said earlier is the single hop communication range. In the simulations we first start sending the information about each individual node throughout the entire region of radius $R$. Then we extend this algorithm for forwarding the aggregated cell information over multiple hops in the tracking region.

\subsection{Algorithm:}

The ultimate goal of a vehicular network system is to have the information of a vehicle by all other vehicles in a tracking region and vice versa. The simplest possible way to implement this is by just forwarding all the nodes information in every transmission cycle of a vehicle. Thus, if a vehicle transmits all $\mathrm{N}$ vehicles information at a frequency of $\mathrm{p}$ times, then the communication rate at each node in unit time should be $\mathrm{O}($ N.p). From the above statement it can be concluded that, as the number of vehicles in the tracking region increases the required communication rate grows large. Also if ' $\mathrm{C}$ ' represents the wireless channel transmission capacity at one node in bits per second then, $\mathrm{p}<\frac{C}{N}$. Thus the transmission frequency should be decreasing as the increase in the number of vehicles in the region. As a result the staleness would be growing more which leads to lesser accuracy in the network.

The solution to these problems is to forward the vehicle information at a rate proportional to the distance from the source vehicle. Also the receiving vehicle doesn't forward that information if it has more updated data from other vehicles. Thus we can have a system with greater update rate, accuracy and lesser communication cost even with the increase in $N$ number. The complete algorithm is given below in the form of <event->action> statements. 
The execution of the algorithm at the vehicle ' $j$ ' starts with the initialization of the variables. The counters and the sequence number are set to zero and the list of vehicles in the region $R$ includes the node $j$ itself. Then the timer starts with the time period of $1 / p$. When the timer is fired then the execution starts with the increase in the sequence number. Then the node $j$ itself is added to the forwarding list of nodes as its own information should be propagated. Then we look for all the vehicles in the region $R$ that we have. If the vehicle ' $i$ ' is at a hop distance equal to the multiples of sequence number, then the node ' $i$ ' is added to the forwarding list of ' $j$ ' satisfying the condition that the information of node ' $i$ ' it is not received from more than one source since ' $j$ 's last transmission. If forwarded, the counter for $i$ 's information is cleared to zero.

On the other hand while receiving the information of every vehicle; the receiving vehicle validates the timestamp of the source information such that it is more updated than the one that it already had. Only if this condition is met the info is updated, otherwise discarded. Also the counter for $i$ 's information is made equal to 1 on every successful update of info. If the node receives the recent update again it just increments the counter for $i$ 's information. Doing so the node doesn't forward that information again as it is already being circulated. If the node receives an older information than it currently have it doesn't increment the counter for i's info. This will make sure that the in the next transmission a much newer version of information is circulated subsiding the older information. In case of the aggregated vehicles information propagation, all the vehicles compute the average of individual vehicles information that they receive from their near neighborhood which is again transmitted in the distance sensitive manner over cells. 


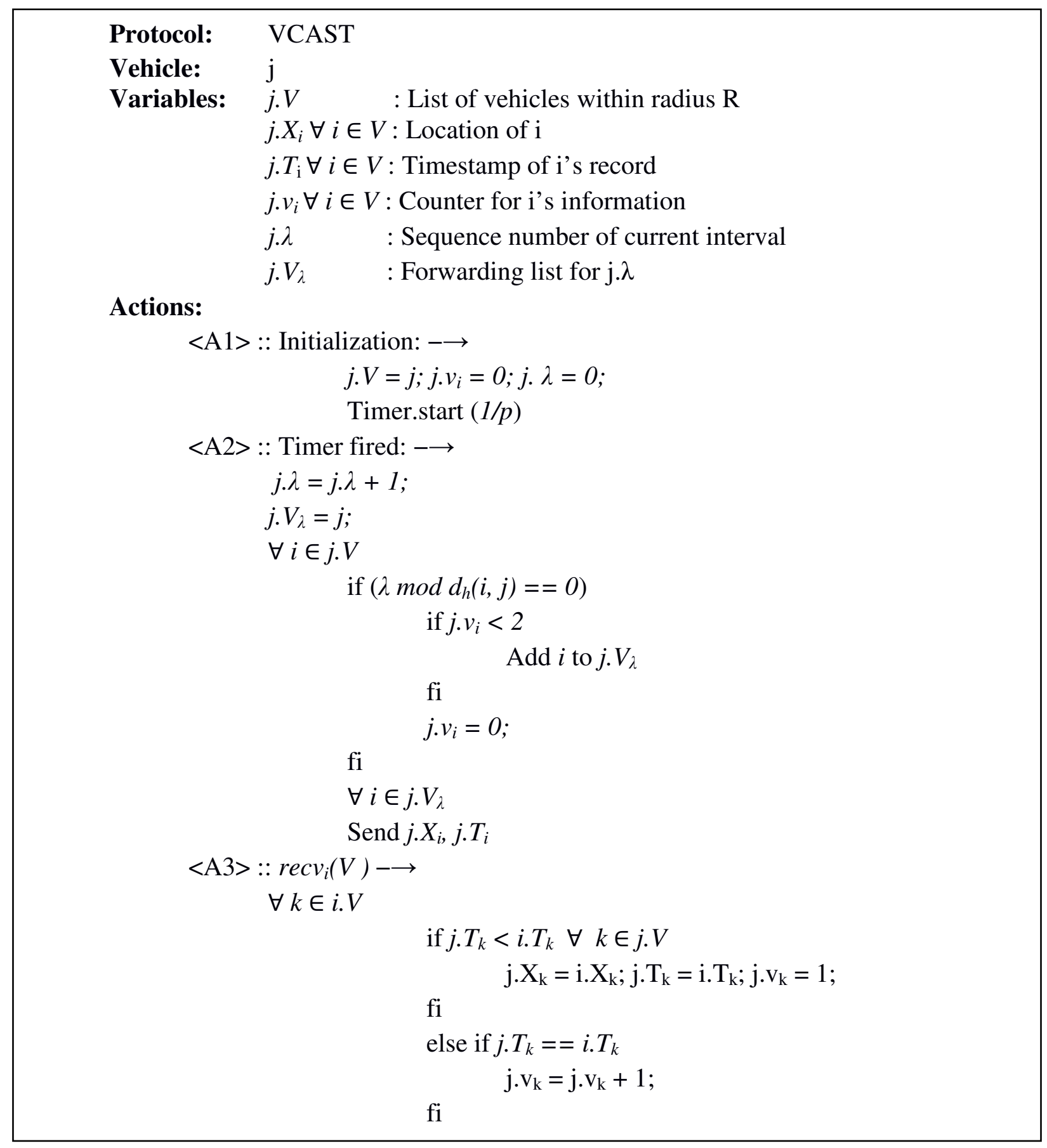

VCAST Algorithm: An infrastructure-less vehicular traffic information service with distancesensitive precision. 


\subsection{Analysis}

Theorem 3.1: The data communication per unit time per each node using VCAST for the exchange of information within the region of distance $R$ from itself is bounded by $O\left(\frac{R p}{r_{h}}\right)$

Proof: Let B denotes the average amount of data communication per unit time per each node with VCAST. Till a distance of at most $h$ communication hops from it, the number of vehicles is bounded by $O\left(\rho h^{2} r_{h}^{2}\right)$. Thus the number of vehicles that are exactly at a distance of h hops is given by $O\left(\rho h r_{h}^{2}\right)$. Also the information at a distance of $h$ hops is broad-casted at every $\frac{p}{h} H z$ and hence the total communication volume about vehicles that are at h hops away is bounded by $O\left(\rho p r_{h}^{2}\right)$. Also note that the information is broad-casted by at most one vehicle in every area of $\pi r_{h}^{2}$. On an average each node is responsible for communicating only $O\left(\frac{\rho p r_{h}^{2}}{\rho r_{h}^{2}}\right)$ bits about vehicles at distance of h hops away from itself. Also remembering that node broadcasts its own state with frequency $\mathrm{p} \mathrm{Hz}$, we sum up over all distances.

$$
B=O\left(p+\sum_{i=1}^{\frac{R}{r_{h}}} \frac{p \rho r_{h}^{2}}{\rho r_{h}^{2}}\right)=O\left(\frac{R p}{r_{h}}\right)
$$

From the formula it can be interpreted that the volume of data communicated per node per unit time does not depend on the vehicle count. Thus, if $C$ denotes the transmission capacity per node then $p<\frac{C r_{h}}{R}$. It can be inferred that the broadcast frequency at the source is not limited by the number of vehicles but on the radius of the tracking zone in terms of communication hops. All these results are interpreted in the ideal case scenario where there is not network contention and hence the information about a node at $k$ communication hops away is broadcasted at most once every $k$ intervals.

\section{Comparison with respect to multi-hop broadcast protocols without distance-sensitivity:}

The case of a broadcast model without distance-sensitivity is similar to the flooding model. In this scenario each node broadcasts information about all nodes in the region at a rate of $p$ times every second yielding a communication cost per node that is bounded by $O\left(\rho p R^{2}\right)$. This shows that the network does not scale with size. Also this would yield to channel contention that reduces the required source broadcast rate of $p$. As discussed in Chapter 2, several heuristics 
were proposed to address this broadcast storm problem so that redundant broadcasts are eliminated. Thus avoiding the redundancy in message forwarding, the average communication cost reduces to a factor of $\rho \pi r_{h}^{2}$ using this naive flooding approach, yielding to an average communication per node of $\mathrm{O}\left(p\left(\frac{R}{r_{h}}\right)^{2}\right)$. Comparing this with the results of Theorem 3.1, we can clearly note that the communication cost per node decreases by a factor of $\frac{R}{r_{h}}$ using the distancesensitive mechanism. This reduction in the communication cost would result in smaller channel contention and allows a higher broadcast rate at smaller distances. This is especially crucial when forwarding information over large areas because it ensures that information supply rates at smaller distances are not compromised for having to forward information over multiple hops.

STALENESS: Staleness is the measurement used in stating the accuracy of the information possessed by a vehicle. Formally this can be defined in the following way,

(Staleness $(j, i))$. The staleness $S(j, i, t)$ in the state of vehicle $i$ as possessed by vehicle $j$ at time $t$ is the time elapsed between two adjacent updates regarding $i$ at $j$ and is given by $S(j, i, t)=,t-j . T_{i}$, where $j . T_{i}$ is the time at which $j$ received information about $i$ previously.

Theorem 3.2: Maximum Staleness at vehicle $j$ regarding the information of vehicle $i$ with VCAST is bounded by $O\left(\frac{d_{h}(i, j)^{2}}{p}\right)$, i.e., $O\left(\frac{d(i, j)^{2}}{p r_{h}^{2}}\right)$

Proof: Let a vehicle at $j$ be $h$ communication hops away from the vehicle at $i$, then the distance between vehicles $i$ and $j$ is given by $\mathrm{d}_{\mathrm{h}}(i, j)=\mathrm{h}$. At the distance of $h-l$ communication hops away from vehicle at $i$, the information about the vehicle $i$ is updated only once every $h-1$ intervals. Similarly at h-2 communication hops away from $i$, information about the vehicle $i$ is updated once every h-2 intervals. Summing this from 1 to $h$ hops would give us the result bounded by $O\left(\frac{d(i, j)^{2}}{p r_{h}^{2}}\right)$. From this derivation it can be inferred that the staleness at a distance doesn't depend on the density of the vehicles or the size of the tracking region, instead it depends on the initial transmission frequency and the communication hop distance. But, contradicting to this as the initial transmission frequency or the communication range increases predominantly, it would yield in more number of collisions in the network that can have an adverse effect on the reliability and system accuracy. 
Theorem 3.3: In case of aggregated cell information propagation using VCAST, the communicated volume of data, per each node, in unit time for fetching the information about the each cell at distance of up to $R$ is bounded by $O\left(\frac{R p}{\rho r_{c} r_{h}^{2}}\right)$.

Proof: Let $\mathrm{B}_{\mathrm{c}}$ is the average amount of data communicated per unit time in the aggregate cell information propagation scenario of VCAST. Previously it has been stated that $d_{c}(i, j)$ denotes the cell distance between vehicle $i$ and cell $j$, i.e., the smaller number of cells traversed to reach cell $j$ from $i$. The number of cells at a cell distance of at most $d_{c}$ from a vehicle is bounded by $O\left(\frac{\rho d_{c}^{2} r_{c}^{2}}{\rho r_{c}^{2}}\right)=O\left(d_{c}^{2}\right)$. As a result, the number of cells at a distance of at most $\mathrm{d}_{\mathrm{c}}$ from a vehicle is bounded by $O\left(d_{c}\right)$. Information about the cell at distance $d_{c}$ away is broadcasted at $\frac{p}{d_{c}} H z$ frequency. Thus the total amount of data to be communicated about cells at distance $d_{c}$ away is $O(p)$. Note that this information is broadcasted by at most one vehicle in every area $\pi r_{h}^{2}$. On the whole, each node is responsible for communication only $O\left(\frac{p}{\rho r_{h}^{2}}\right)$ bits about cells at distance $d_{c}$ away from itself. Aggregating the entire process over the cell distances of $d_{c}=1$ to $d_{c}=\frac{R}{r_{c}}$, we get:

$$
B_{c}=O\left(\sum_{i=1}^{\frac{R}{r_{c}}} \frac{R p}{\rho r_{h}^{2}}\right)=O\left(\frac{R p}{r_{c} r_{h}^{2}}\right)
$$

Comparing this with the result from Theorem 3.1, we note that the average communication cost reduces by a factor equal to the number of vehicles in each cell $\left(\rho r_{c} r_{h}\right)$ because only aggregate information about the cell is propagated with distance-sensitive rate as opposed to propagating information about each vehicle in the cell.

Theorem 3.4: The staleness of the cell $z$ at the vehicle $j$ using VCAST, when aggregation of information is forwarded is bounded by $O\left(\frac{d_{c}(j, z) d_{h}(j, z)}{p}\right)$, where $d_{h}(j, z)$ is the communication hop distance between $j$ and closest vehicle to $j$ in cell $z$.

Proof: Considering a vehicle $j$ and cell $z$, the lowest broadcast rate about cell $z$ that is available for vehicle $j$ is $\frac{d_{c}(j, z)}{p}$. Also the number of communication hops between $j$ and closest vehicle in $z$ is $d_{h}(j, z)$. Hence, latency in forwarding information about cell $z$ to vehicle $j$ is 
bounded by $\frac{d_{c}(j, z) d_{h}(j, z)}{p}$. Also note that the subsequent update about cell $z$ will be available within $\frac{d_{c}(j, z)}{p}$ seconds. 


\section{Chapter 4}

\section{Network Simulator-3 (NS3)}

Testing the algorithm for vehicular networks with real time experiments is quite difficult and would be very expensive setup. In order to overcome these difficulties for testing, network simulators are used. Simulating the real time situations gives us the approximated outputs on these simulators. The VCAST algorithm is tested on Network Simulator 3. The simulator is provided with a set of predefined communication protocols, mobility models and transmission models that generate the real time parameters. Using models and protocols like these, the vehicular nodes are generated and the algorithm is implemented on the system. The output of performance of the algorithm is measured in staleness of the information at the receiving node. Staleness represents the freshness or the accuracy of the information of a vehicle at the receiving vehicle with respect to time. The simulators are quite easy to handle in simulating for different parameters by easily changing the transmission distances, the cell areas, density of the nodes and the size of the region, etc., which cannot be made that easily in the case of a real time experiment.

Network Simulator-3, popularly known as NS3 is a discrete-event network simulator. It was mainly targeted for the use with research and education and set to be an open-source project. The Network Simulator 3 was completely developed in $\mathrm{C}++$, along with the optional python bindings. Thus the Network Simulation script can be written either in $\mathrm{C}++$ or Python. Using this network simulator one can simulate a network as big as the size of internet. The network simulator 3 is quite easy to handle and maintain. The NS-3 works on a Linux or Linux-like environment. It uses the tools from GNU “toolchain” like gcc, GNU binutils, and gdb.

\subsection{Basic elements of Network Simulator-3}

\subsubsection{Node:}

Node is the generic term used for referring to a computing device that is connected in a network. More precisely, in the case of internet, we call it a host or endsystem. But in NS-3 basic computing abstraction is called as a node that was written with 
the name 'Node' class in $\mathrm{C}++$. This class enables us to manage the representation of these computing devices using methods. One needs to add an application, protocol stack and peripheral cards in order for the computer to work completely in network topography.

\subsubsection{Application:}

The computer software is the one that organizes and manipulates all the resources that are available with it. The classification of software into two categories tells the level of its manipulation. A System Software usually organizes computer resources like processor, memory, disks, network, etc. But these System Software's are not directly helpful to the user. Instead a user runs application that makes use of the system software in order to meet its goals.

In the NS-3 world there is nothing said to be a System Software managing the resources and system calls, but there are Applications that run on the nodes for carrying forward the simulations.

The user making an abstract programing for some event or activity that needs to be done by the nodes in a network is called Application. The class 'Application' is the abstraction for this program. This Application class has methods that are used in managing the representation of various user-level applications.

\subsubsection{Channel:}

Channel is the media over which information is passed from one point (node here) to another point. In the NS-3 simulator, each node is connected to an object representing a communication channel. The Abstraction of this communication channel is represented by the class Channel in $\mathrm{C}++$. This class provides us methods for managing the communication channels that are connected to nodes. We can either simulate a channel with a simple ethernet cable or a switch or a huge open area with obstructions for a wireless network.

\subsubsection{Net Device:}


To connect a node to the channel we need to have some hardware that converts the digital data into analog or optical data that travels through the channel. Such a hardware is called as a peripheral card, installed on the computer. Since the channels vary, there are different set of hardware devices for different types of channels. As these peripheral card implements different network functions, they are called Network Interface Cards (NIC's).

Every hardware needs some drivers for its working and controlling, similarly, network devices need network device drivers collectively called as Net Devices in NS-3. Thus Net Device covers both the driver software and simulated hardware. Thus a Net Device needs to be connected to a node for it to communicate with other nodes through some channel. There may be multiple numbers of Net Devices in the case if a node connects it to many other nodes. The abstraction of this is written in the 'NetDevice' class in C++.

Apart from these basic elements there are many different classes that have been used for the VCAST simulation. These include PositionAllocators, MobilityModels, CallBack Procedures, Graphing tools, etc., discussed in the next section of this chapter.

\subsection{Required Classes}

\subsubsection{Topology Helper Class:}

For a user to simulate a large network, it becomes hard to manage the nodes in assigning the Applications, Communication Channels, Net Devices, Mobility Models, IPaddresses etc., as a number of different operations needed to be performed in linking these elements. To make things easier the Helper classes are used. These helper classes assign all nodes with an abstraction class of the required basic elements and performs operations that are needed for setting up a simulated network.

\subsubsection{Node Container:}

As the name indicates, NodeContainer holds a set of nodes that can be referred directly as a single entity instead of looping for all the required set of nodes. Whenever the nodes are separated in groups and each group is intended to do a different task or to have a different transmission mechanism from the other, the nodes are assigned with different NodeContainer entities. Thus groups of nodes can be easily managed and organized. The 
NodeContainer class written in $\mathrm{C}++$ has a set of methods that makes the user to manage the groups of nodes.

\subsubsection{Mobility Model:}

Mobility models are very much helpful in the case of moving node system in a wireless networking environment. As we know that the nodes in a wireless network model are not fixed, the networking characteristics do keep changing. To track these change in the Network parameters the nodes needed to be simulated with motion. The 'MobilityModel' class written in $\mathrm{C}++$ has set of methods that help us in positioning the nodes and also moving them with respect to time. There are different types of mobility models available in NS-3 like the 'Random2DWalkMobilityModel', '2DWaypointMobilityModel', 'ConstantVelocityMobilityModel', etc. The mobility models are also defined for the 3dimentional motion of nodes which are not used in our scenario of VCAST.

\subsubsection{GNUPlot:}

GNUPlot is a portable command-line driven graphing utility for Linux, OS/2, MS GNU Plot Windows, OSX, VMS, and many other platforms. The GNUPlot tools are used for drawing graphs of staleness as a function of distance in the VCAST. The GNUplot generates a '.plt' file as the output when the simulation is completed. Using this '.plt' file, the GNUplot library for Linux generates the pictorial diagram of the result set.

\subsection{Modules for VCAST Simulation}

There involves three modules that govern the entire operations of simulation for the VCAST algorithm, the primary module or the base module, Vanet Application Module, Vanet Nodes Info module and the Vanet Payload module. The functionality of each of these modules is given below.

\subsubsection{Primary Module/Base Module:}

The primary module is the one which has the main() function and controls the simulation parameters like the time, number of nodes, communication model, mobility 
model, Internet stack, etc. The primary module begins with enabling the Log Components that gives different log information regarding various modules on screen during the simulation. Then the nodes are created and are configured in required positions using different Position Allocator Classes. To these nodes we add different mobility models as per the requirement. We assigned a 'Random 2D Walk' model in this case of VCAST making the nodes move only in one direction on a four lane highway at different speeds. This is followed out by assigning the network device, the channel and the IP addresses. Each of these assignments is configured with the required settings. The 'NetDevice' that we used here is the $802.11 \mathrm{p}$ wireless device set to the default or standard configuration. The physical layer has the same properties that were defined in the YansWifiPHY model with receiver gain set to $0 \mathrm{dBm}$.

The Wifi channel has been implemented from the YansWifiChannel where the Propagation Delay is set to the Constant Speed Propagation Delay model in which the message is transmitted at the same rate in all directions. Also the propagation loss model set here is the 'Range Propagation Loss Model' in which the strength of the signal is dropped depending on the distance from the transmission. Here we defined the maximum transmission range capability of each of the nodes. In the case of fading model we use the 'ThreeLogDistanceLossModel' along with 'NakagamiLossModel'.

This is followed by setting up the MAC layer for all the net devices that we have assigned to the nodes. These MAC addresses are automatically assigned by using the 'NqosWifiMacHelper' class set for default settings using the method 'Default'. Now we assign the RemoteStationManager, which hold a list of per-remote-station state. The remote station manager is a 'ConstantRateWifiManager' which has the constant transmission rate or delay.

Finally the NetDevice, WifiPhy, and the WifiMac are linked to each of the nodes. Then the nodes are assigned with the IP addresses. The base IP address is '129.0.0.0' with the subnet mask of '255.0.0.0'. By doing so, the data can be transmitted from one node to another by referring to the IP addresses in the upper layers of the OSI model. 
Next to it is assigning the Application Module for the nodes. The Application Module is the top layer of the OSI model, i.e., the application layer functionality is defined in this Application Module. We then assign the times at which the application module needs to start after simulation and stop. Then the Run() method of simulation class is called, which is destroyed after the run time specified.

At the end of the simulation the graphing section comes into play where the data from the simulation is collected and is set into the '.plt' file using the 'GNUplot' library. This text file representing the graph data is converted to graphical representation by using the 'gnuplot' command at the terminal.

\subsubsection{Application Module:}

The application module implements the upper most layers or the application layer of the OSI model. A custom application module in NS3 inherits the Application Class. This involve the methods like DoStart(), DoDispose(), StartApplication(), StopApplication(), etc. The StartApplication() method is called at the time specified by Start, and the StopApplication() is called at the time specified by Stop. The other user defined methods include receivingMsg(), SendPacket(), CreatePacket(), etc. In the StartApplication() module we start with the initialization of the socket with a socket number and an 'Receive Any' (0.0.0.0) ip-address assigned to it. Then a timer is started that counts any number randomly from $90 \%$ to $110 \%$ of the transmission frequency. By doing so we ensure that less number of transmission collisions happens.

When the timer gets fired, the method SendPacket() is called back which calls another method, createpacket() that creates the data packet, and sends the data packet. This again restarts the timer. In the createpacket() method, first the data of the own node is added to the packet that is queued for sending followed by the other nodes forwarded data depending on the distances. We iterate through all the nodes and find only the ones that have a hop distance in multiple of the TurnNumber. Also we make sure that the information about the same node is not received from more than a single source since its last transmission, so that redundant transmissions of same data is eliminated. 
On the other hand, receivingMsg() method is called whenever there is a message received at the assigned socket. The node then iterates through all the nodes data in the received data packet and evaluates it such that if the received info is updated version from what it already had, the node saves that information, otherwise discards it. Before doing so it updates the staleness regarding that particular node information at the receiving node. Also the redundancy counter gets incremented.

Each of the nodes has the same functionality that will be running individually with respect to one another. The simulator takes care of the execution of this application in all the nodes independent to each other.

\subsubsection{NodeInfo Module:}

The NodeInfo module synchronizes the data of all the independent nodes in the network together for the aggregation of the node information like the staleness and the actual distances with respect to each other for the simulation aspect only. The methods in NodeInfo module includes methods like 'updateTotalStaleness', 'getTotalStaleness', 'updateActualDistance', 'getActualDistance', 'getNeighbors'. The 'updateTotalStaleness' and 'getTotalStaleness' methods are called from the VanetApplication module, such that the individual staleness information from each of the nodes is collected and aggregated. The next two methods 'updateActualDistance' and 'getActualDistance' measures and returns the actual distance between the two nodes as this may not be the distance known by the nodes from the information it received as nodes keep moving in and out the tracking region. The 'getNeighbor' function returns the node ID's of all the neighboring nodes by calculating its actual distance and the communication range.

\subsubsection{Vanet Payload Module:}

The Vanet Payload Module is the payload template for the information that needs to be exchanged. This includes the fields' node_ID, TimeStamp, Position_X, Position_Y, Velocity, etc. When the data packet is created it takes the template from the Payload module and puts the data into the Payload object with the corresponding fields. The fields in the VanetPayload Module can further be incremented. As the number of fields in the 
payload increases the packet data size increases. This leads to the additional transmission time because of the increase in the data packet size. At high frequency of data transmission the higher transmission times would cause the problem of increased collision rates in the network. 


\section{Chapter 5}

\section{Results \& Observations}

Evaluating the VCAST performance is carried out with various experimental setups using ns-3. This involves different mobility models, transmission distances and different transmission frequencies. We use IEEE 802.11p physical layer communication model working at 5.9G $\mathrm{Hz}$ with a $10 \mathrm{MHz}$ bandwidth, thus emulating the physical layer characteristics of Dedicated Short Range Communication devices. Also it was assumed that the nodes transmit at a speed of $3 \mathrm{Mbps}$ over the network. The highway is extended to a length of $5 \mathrm{kms}$ (5000meters) occupied by 800 vehicles and each vehicle is on an average of $25 \mathrm{~m}, 15 \mathrm{~m}$ and 10 meters apart from one another. There are 800 vehicles on a whole, aligned on the highway. The communication range for these nodes (vehicles) were choosen to be $200 \mathrm{~m}, 300 \mathrm{~m}, 400 \mathrm{~m}$ and $500 \mathrm{~m}$ and the broadcast rates of these are $20 \mathrm{~Hz}, 10 \mathrm{~Hz}, 5 \mathrm{~Hz}, 2.5 \mathrm{~Hz}$. These were in the range of expected transmission rate and range values of Here I am messages for intelligent transportation systems. At each vehicle, the staleness regarding each other vehicle was measured on every successful reception of information. In the case of aggregation model we propagate the information regarding a cell instead of individual vehicle information and measure the maximum staleness with respect to every other cell. We then group the maximum staleness based on pairwise distances between vehicle-vehicle and vehicle-cell respectively. The average of these measurements over multiple experiments is used in evaluations.

- The first experimental setup starts with a uniform velocity model in which the average intervehicular separation is maintained at $25 \mathrm{~m}$ throughout the duration of the simulation. By simulating uniform velocity, the relative distance between vehicles is maintained during the course of the simulation allowing the clear characterization of distance versus information staleness. In these simulations, to systematically study the impact of broadcast range on system performance, we disable the channel fading and instead use fixed radius communication.

- Next we enable mobility on vehicles so that the inter-vehicular separation varies over simulation time. These vehicles move at random speeds specified in bounds. The outputs of 
these simulations are affected by the varying or dynamic inter-vehicular distances because of the behavior of forwarding vehicular information in VCAST at variable densities.

- After incorporating mobility we add channel fading models for evaluations. We use the Nakagami fading model in which the parameters are set in such a way that at smaller distances $(<30 \mathrm{~m})$, Rician distribution was followed, at intermediate distances (between 30m and $100 \mathrm{~m})$ Rayleigh model and finally pre-Rayleigh model at higher distances $(>100 \mathrm{~m})$ is followed [19]. We evaluate the performances at two different power levels.

- After the first three techniques the fourth one is to study the effect of density by considering the average inter-vehicular distances of $10 \mathrm{~m}$ and $15 \mathrm{~m}$.

- Then we evaluate the communication cost at each node in terms of number of messages transmitted per second. We specifically highlight the uniformity in the distribution of the communication load across different nodes.

- Finally, we quantify the impact of information aggregation on staleness.

\subsection{Fixed inter-vehicular separation}

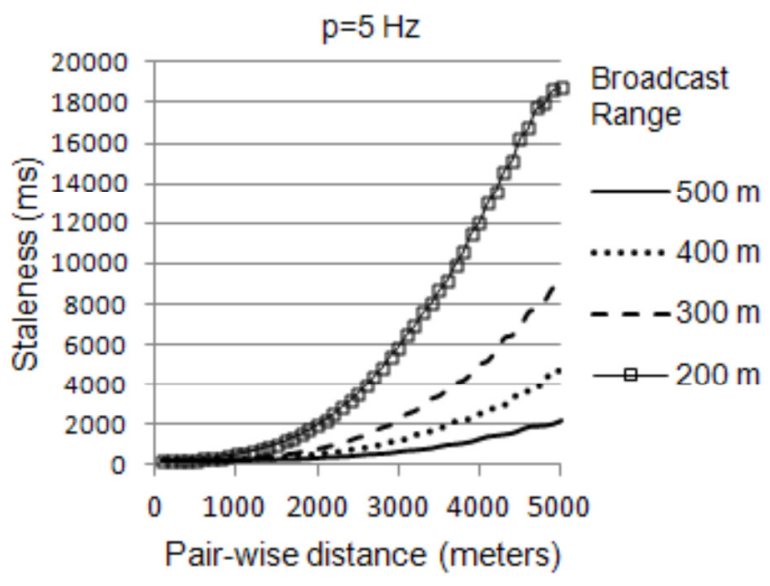

Fig 5.1(a): Maximum staleness vs. pair-wise vehicular distance: $p=5 \mathrm{~Hz}$, one hop communication range of $200 \mathrm{~m}, 300 \mathrm{~m}, 400 \mathrm{~m}$ and $500 \mathrm{~m}$.

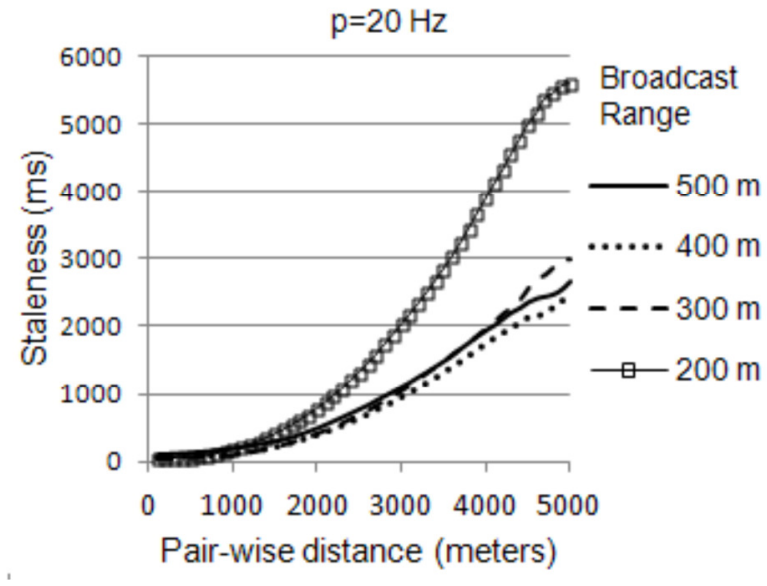

Fig 5.1(b): Maximum staleness vs. pair-wise vehicular distance: $p=20 \mathrm{~Hz}$, one hop communication range of 200m, 300m, $400 \mathrm{~m}$ and $500 \mathrm{~m}$. 
This section majorly concentrates on the performance of the VCAST protocol in a fixed inter-vehicular separation model without any channel fading effects. The 800 vehicles are placed in such a way that the average-separation is $25 \mathrm{~m}$ along the 4 lane highway. Maintaining the relative distance between vehicles during the course of simulation allows a clear characterization of distance versus information staleness. Using fixed range propagation loss model would help us in study the effect of communication range on system performance.

In Fig. 5.1(a) and Fig. 5.1(b) we plot the maximum staleness in vehicle information with respect to the inter-vehicular distances for transmission frequency of $\mathrm{p}=5 \mathrm{~Hz}$ and $\mathrm{p}=20 \mathrm{~Hz}$ respectively at different communication ranges. In case of $\mathrm{p}=5 \mathrm{~Hz}$ we can observe that the staleness increases at larger distances as the communication range decreases since the information needs to travel more number of hops. On the other had when $\mathrm{p}=20 \mathrm{~Hz}$, the staleness with $500 \mathrm{~m}$ communication range exhibits higher staleness than a communication range of $400 \mathrm{~m}$. This is because of the channel contention and hidden node problems caused by combination of high source broadcast rate and high communication range.

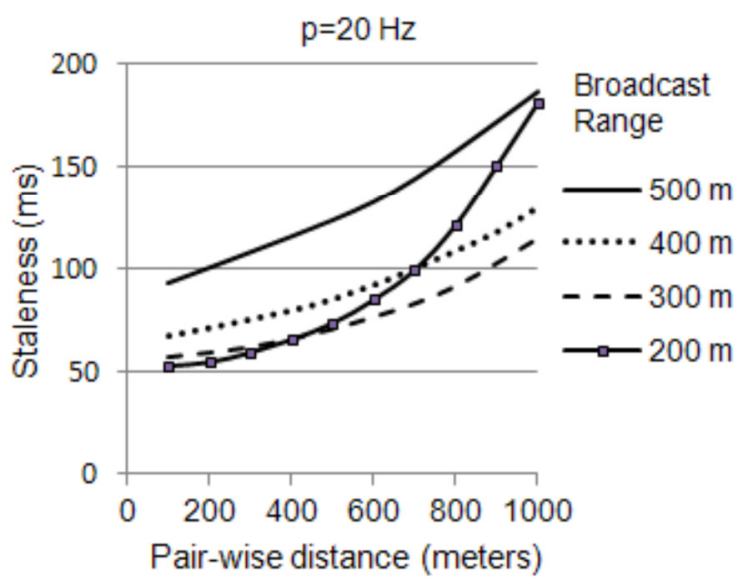

Fig 5.2: Maximum staleness vs. pair-wise vehicular distance: $p=20 \mathrm{~Hz}$ at smaller distances (Zoomed-in version of Fig. 5.1(b))

Fig. 5.2, which is the zoomed version of Fig 5.1(b), shows the staleness at closer distances with $\mathrm{p}=20 \mathrm{~Hz}$ for different communication ranges. It is seen that, increasing the communication range would have adverse results on information accuracy at smaller distances. Using the distance sensitive mechanism the communication distance can be kept low and thus decreasing the staleness at smaller distances while progressively increasing the staleness at higher distances. It can be observed that at $100 \mathrm{~m}$ of inter vehicular separation the staleness drops 
to half with respect to the communication range of $500 \mathrm{~m}$ and $200 \mathrm{~m}$. With single-hop broadcasting techniques, a higher range would yield higher staleness even for smaller distances while a smaller range would prevent information supply beyond that distance.

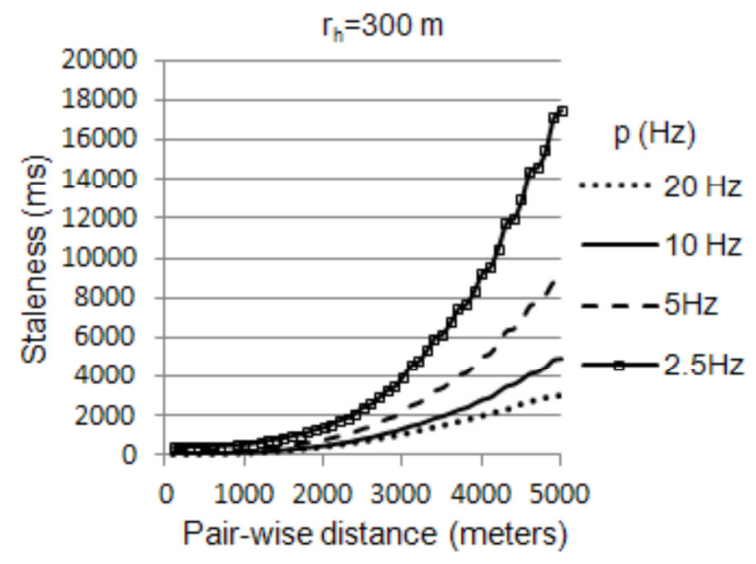

Fig 5.3(a): Maximum staleness vs. pair-wise vehicular distance: $r_{h}=300 \mathrm{~m}$

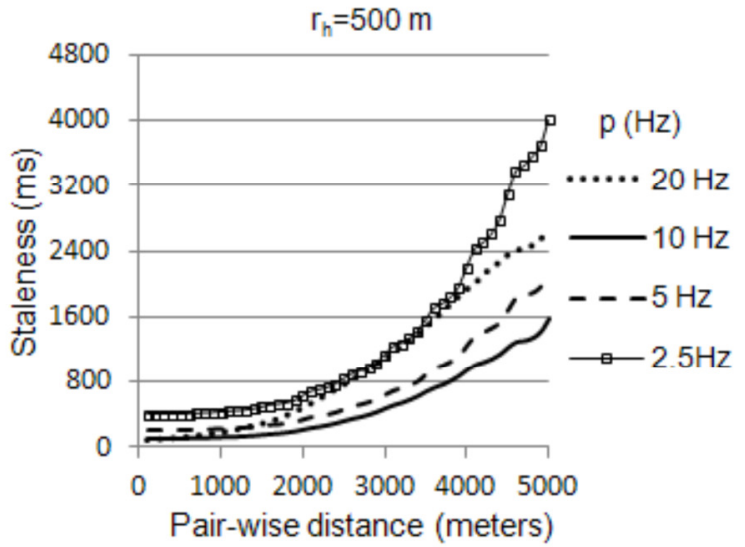

Fig 5.3(b): Maximum staleness vs. pair-wise vehicular distance: $r h=500 \mathrm{~m}$

Using Fig. 5.3(a) \& Fig. 5.3(b), we now investigate the effect of initial broadcast rate on the performance of the system. The staleness at different values of $\mathrm{p}$ for $r_{h}=300 \mathrm{~m}$ and $r_{h}=500 \mathrm{~m}$ are compared. We notice that at $r_{h}=300 \mathrm{~m}$, a higher broadcast rate is able to decrease the staleness significantly at higher distances. On the other hand at $r_{h}=500 \mathrm{~m}$ and broadcast rate of $p=20 \mathrm{~Hz}$ outputs noisome results in information precision because of increased contention.

\subsection{Impact of time varying inter-vehicular separations}

In this set of experiments the vehicles move with different speeds along the same lane on the four-lane highway. The vehicles are initially placed at a separation of $25 \mathrm{~m}$ all along a highway of $5 \mathrm{Km}$. As the vehicles start moving, the distance between the neighboring vehicles in same lane varies between $1 \mathrm{~m}$ and $49 \mathrm{~m}$ as each of the vehicles choose a random speed of Gaussian Normal Distribution. This affects the density as well as the forwarding rules in VCAST. Note that the forwarding of vehicular information in any interval is based on the current knowledge about the vehicle's location and thus the choice of a forwarding rate for a vehicle's state is based on stale information. Fig. 5.4(a) and Fig. 5.4(b) shows the impact of time-varying separation at $p=5 \mathrm{~Hz}$ and $p=20 \mathrm{~Hz}$ respectively. Comparing this with the $25 \mathrm{~m}$ inter vehicular distance scenario from Fig. 5.1, it can be inferred that the staleness at $p=5 \mathrm{~Hz}$ are relatively 


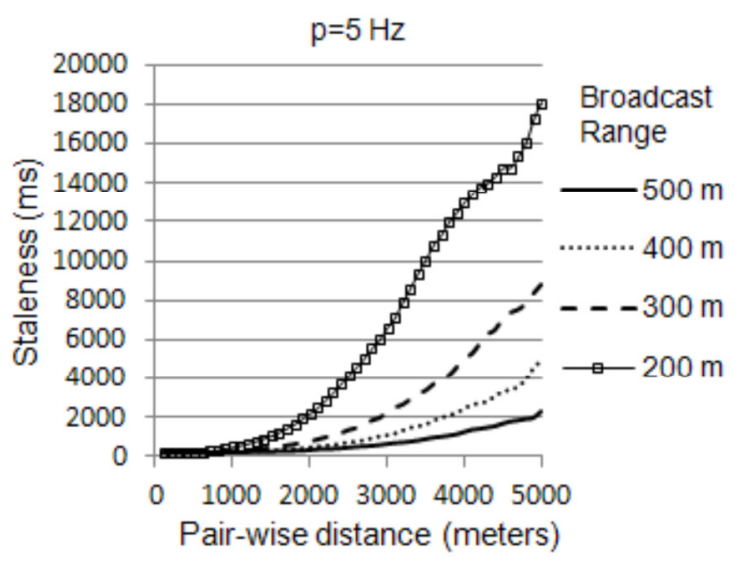

Fig. 5.4(a): Maximum staleness vs pair-wise vehicular distance: $p=5 \mathrm{~Hz}$

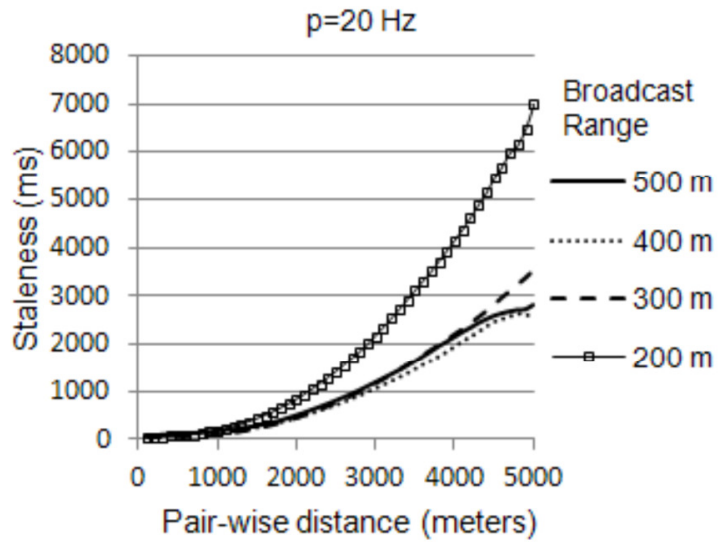

Fig. 5.4 (b): Maximum staleness vs pair-wise vehicular distance: $p=20 \mathrm{~Hz}$

Fig. 5.4: Impact of time-varying vehicular separations.

unaffected by time varying separations, where as a slight increase is observed at $p=20 \mathrm{~Hz}$, especially with smaller communication range values which may be of the dis-connectivity in communication between vehicles.

\subsection{Impact of fading in conjunction with variable speed}

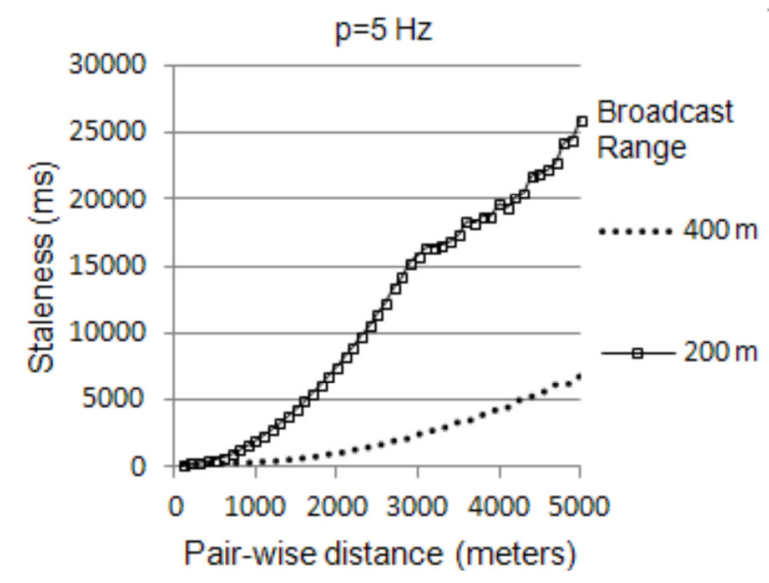

Fig. 5.5(a): Maximum staleness vs. pair-wise vehicular distance: $p=5 \mathrm{~Hz}$

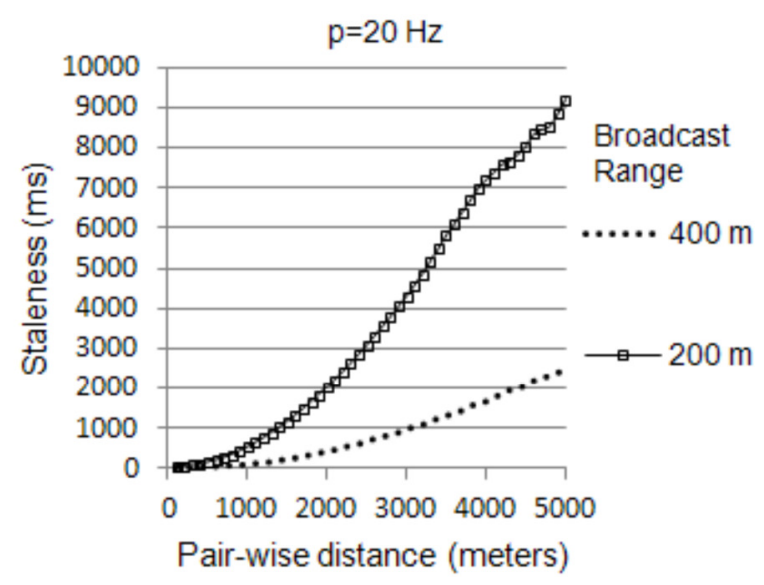

Fig. 5.5(b): Maximum staleness vs. pair-wise vehicular distance: $p=20 \mathrm{~Hz}$

Fig. 5.5: Impact of channel fading.

Along with the varying density using mobility we incorporate the channel fading in the VCAST simulations. The fading model is implemented according to the specified characteristics by recent papers, stating to follow Rician distribution at smaller distances $(<30 \mathrm{~m})$, and Raleigh model at intermediate distances (between 30m and 100m) and finally pre-Rayleigh model at 
higher distances $(>100 \mathrm{~m})$. We choose the transmission power levels to be $16 \mathrm{~dB}$ and $20 \mathrm{~dB}$ and make the receiver sensitivity of $-64 \mathrm{~dB}$ which is expected to deliver a maximum communication range of $200 \mathrm{~m}$ and $400 \mathrm{~m}$ respectively. Fig. 5.5(a) and Fig. 5.5(b) shows the impact of fading at $\mathrm{p}=5 \mathrm{~Hz}$ and $\mathrm{p}=20 \mathrm{~Hz}$ and we compare these results to the no fading situation in Fig. 5.4. We can see a significant increase in the information staleness particularly at smaller communication range i.e., at $200 \mathrm{~m}$, because the effective communication range in the case of fading drops to $100 \mathrm{~m}$. Hence the number of hops in the network increases leading to increase in the staleness.

\subsection{Impact of density}

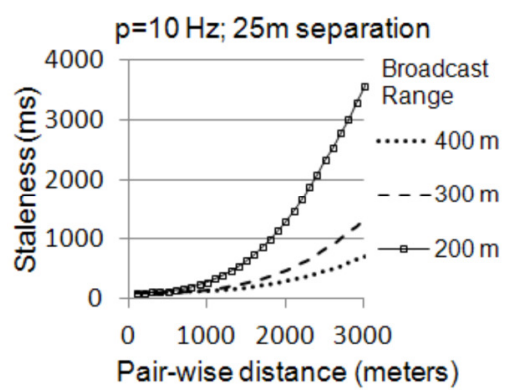

Fig. 5.6(a): Maximum Staleness vs. pair-wise vehicular distance: $p=10 \mathrm{~Hz}$ and average separation $25 m$

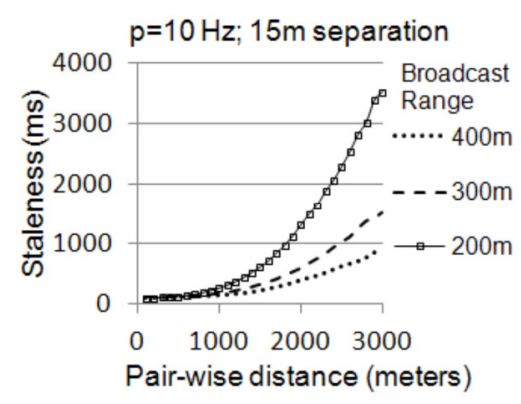

Fig. 5.6(b): Maximum Staleness vs. pair-wise vehicular distance: $p=10 \mathrm{~Hz}$ and average separation $15 \mathrm{~m}$

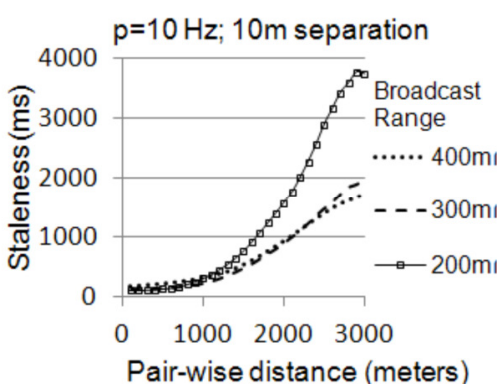

Fig. 5.6(c): Maximum Staleness vs. pair-wise vehicular distance: $p=10 \mathrm{~Hz}$ and average separation $10 m$

Fig. 5.6: Impact of network density.

In order to validate the performance of the system at different densities, we simulate the VCAST technique with average inter-vehicular separation of $15 \mathrm{~m}$ and 10m. Fig. 5.6(a), Fig. 5.6(b) and Fig. 5.6(c) shows the results for average separations of $25 \mathrm{~m}, 15 \mathrm{~m}$ and $10 \mathrm{~m}$ respectively. We retain the same number of vehicles (800) in all the simulations. Thus the vehicles now span to a smaller region, thus showing staleness up to a maximum inter-vehicular distance of $3 \mathrm{Km}$ only. The result graphs shows that the staleness is mostly unaffected by vehicular density variations. Only a small increase in staleness is observed at the density of $10 \mathrm{~m}$ average separation with a relatively high communication range of $r_{h}=400 \mathrm{~m}$. This is because of higher contention at higher density conditions. 


\subsection{Analysis of communication cost}

The communication cost by each node using VCAST for forwarding vehicular information is analyzed. We calculate the average number of records forwarded by each node per second, as the metric for communication cost. From the Theorem 3.1, it is stated that the average

communication cost per node is bounded by $\mathrm{O}\left(\frac{R p}{r_{h}}\right)$, where $p$ is the source broadcast rate, $R$ is the network radius and $r_{h}$ is the single hop communication range. According to the theorem, the communication cost should be decreasing with the increase in the communication range as the total vehicular information broadcasting is shared by larger number of nodes in each communication zone. However, these results assume that information about nodes at a distance of $k$ communication hops is broadcasted at most once every $k$ intervals in each communication neighborhood. We first show this ideal scenario by evaluating the communication cost using the contention-free (TDMA) slots for each vehicle for transmission in every interval. In Fig. 5.7(a), showing the ideal scenario of the VCAST system, the communication cost decreases with increase in the communication range.

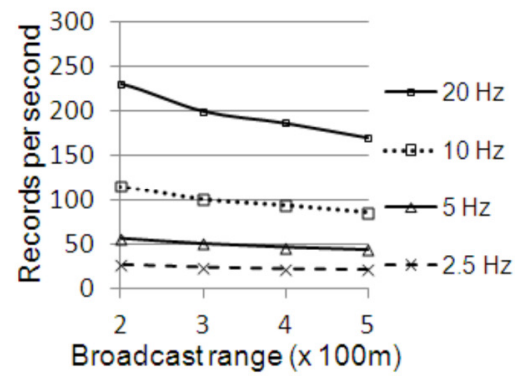

Fig 5.7(a): Simulation with no channel contention

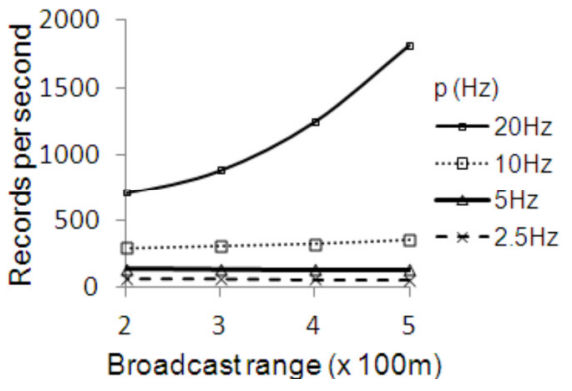

Fig 5.7(b): Simulation with channel contention

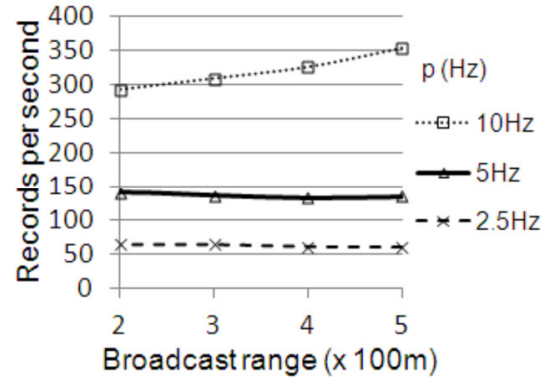

Fig 5.7(c): Zoomed version of (b) for $p=10 \mathrm{~Hz}, p=5 \mathrm{~Hz}$ and $p=2.5 \mathrm{~Hz}$

Fig 5.7: Analysis of communication cost. Denstiy $=25 \mathrm{~m}$ separation.

But considering the practical situations, we simulate the 802.11 random access MAC model with channel contention and hidden terminal problem resulting in the vehicular records to be transmitted multiple times resulting in higher communication cost. It can be noted from the Fig 5.7(b) that, the average communication cost at broadcast frequency rates of $2.5 \mathrm{~Hz}, 5 \mathrm{~Hz}$ and $10 \mathrm{~Hz}$ with contention, is about 2-4 times higher than the corresponding values without channel contention. From the zoomed version of Fig. 5.7(b) shown in Fig. 5.7(c), it can be noticed that 
the communication cost increases with increase in communication range with channel contention in consideration. At the source broadcast rate of $20 \mathrm{~Hz}$ it can be inferred that the communication cost increases randomly because of the extreme parameters.

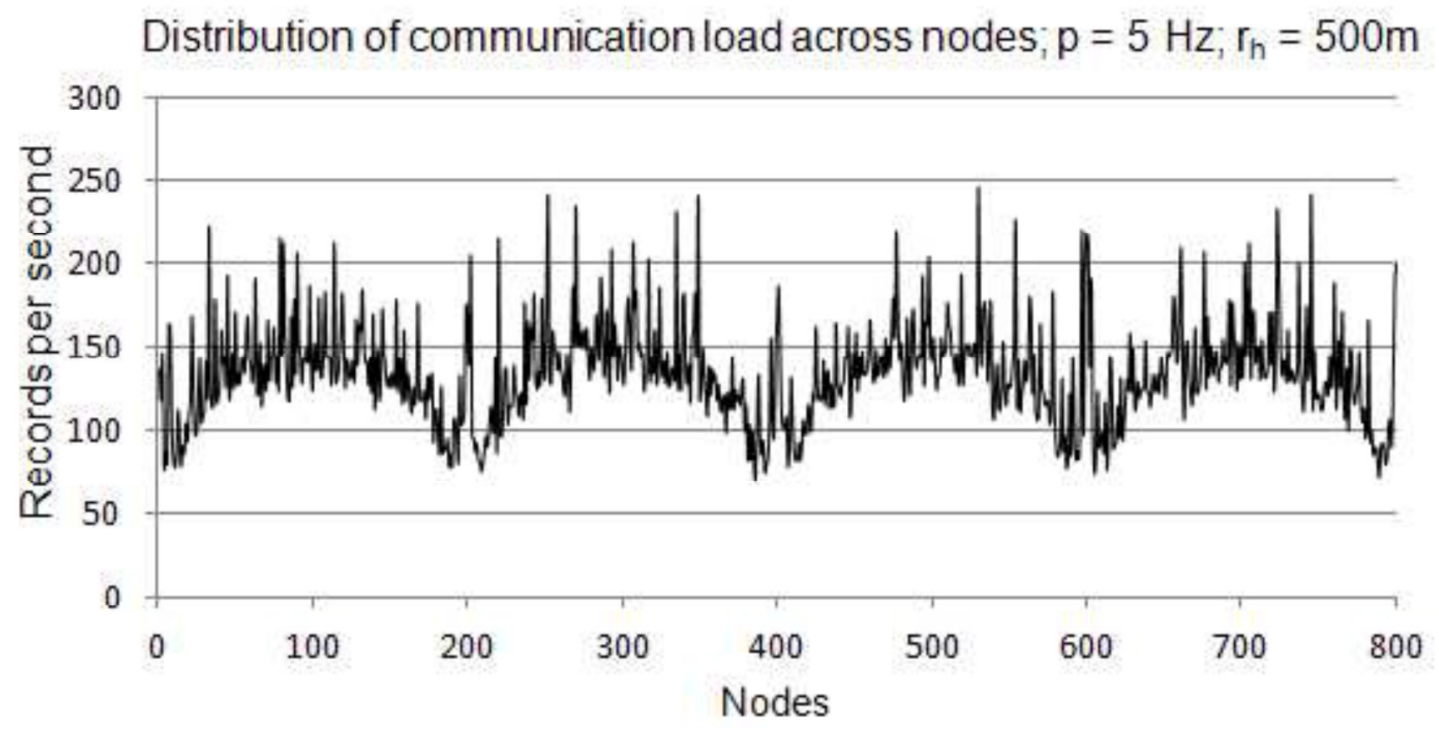

Fig 5.8: Distribution of communication load across vehicles in the network; $p=5 \mathrm{~Hz}, r_{h}=500 \mathrm{~m}$

Fig. 5.8, shows the uniformity in the communication costs across different vehicles by using a scatter plot of average communication cost for each of the 800 vehicles. The results shown in the figure are for the broadcast rate of $5 \mathrm{~Hz}$ and communication range of $500 \mathrm{~m}$ for an inter-vehicular separation of $25 \mathrm{~m}$. It can be observed that the load sharing is achieved with lesser variance without the use of any underlying clustering and scheduling structure. This shows that the lesser number of nodes are not being loaded with forwarding information about a larger number of nodes. Ensuring that the load is balanced over all the nodes, it can be said that fairness is achieved without making the vehicles starve in transmitting their own information - which is an important factor for making distance sensitivity in information staleness. The periodic dips in the communication load are because of the lesser number of neighbors for vehicles at the edge of the network.

\subsection{Impact of aggregation}

In this section, we deal with VCAST simulations in which aggregate information about a cell is transmitted over multiple hops as opposed to individual vehicle information. The cells are 
of equal sized obtained by dividing the region of simulation and the width of these cells is assumed to be equal to the single hop communication range.

Fig. 5.9 shows the average records transmitted by each node per second when the aggregated information is propagated instead of individual vehicle information. The data is shown for $p=5 \mathrm{~Hz}$ and $p=20 \mathrm{~Hz}$ at $r_{h}=200 \mathrm{~m}$ and $r_{h}=500 \mathrm{~m}$. Comparing to Fig. 5.7(b), it can be inferred that the total data communicated per second is reduced significantly even with $p=20 H z$. This is because of two reasons - firstly aggregate data is transmitted as opposed to individual vehicle data and secondly the lower data volume reduces channel contention and suppresses duplicate transmissions.

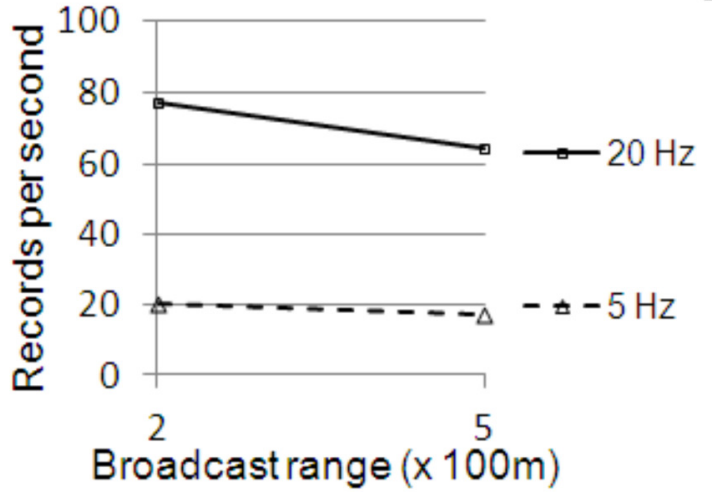

Fig 5.9: Analysis of communication cost

Fig. 5.10 shows the plot of maximum staleness in aggregate cell information against

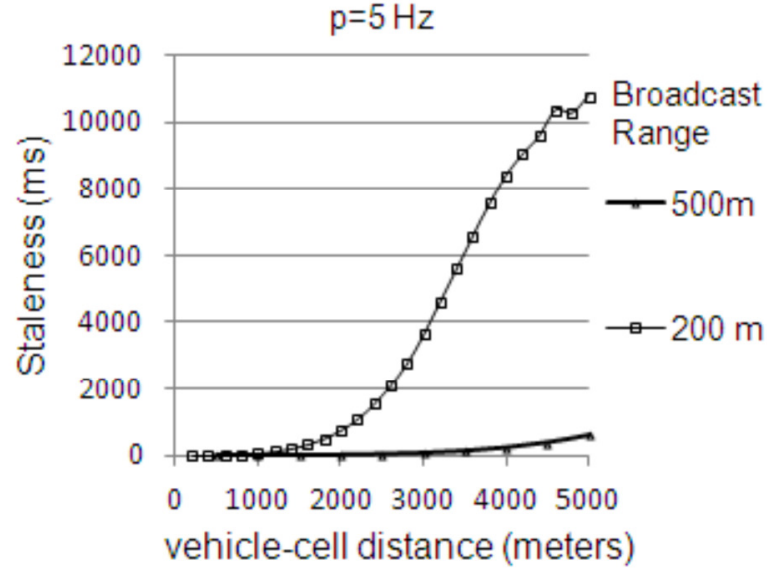

Fig. 5.10(a): Maximum staleness vs vehicle-cell distance: $p=5 \mathrm{~Hz}$

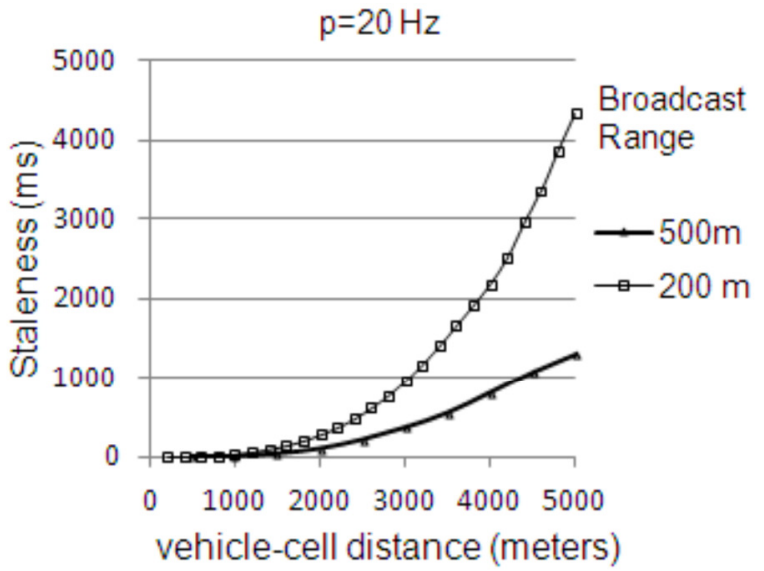

Fig. 5.10(b): Maximum staleness vs vehicle-cell distance: $p=20 \mathrm{~Hz}$

Fig. 5.10: Impact of information aggregation.

pairwise vehicle-cell distance for $p=5 \mathrm{~Hz}$ and $p=20 \mathrm{~Hz}$ at $r_{h}=200 \mathrm{~m}$ and $r_{h}=500 \mathrm{~m}$. The average inter-vehicular distance between them is not varied throughout the simulation and is equal to 25m. Comparing the results with Fig 5.1(a) of uniform velocity of individual vehicular information propagation, it can be seen that the information staleness is significantly lower at corresponding distances with aggregation because a single message from a cell is enough to 
convey the information about the entire cell as opposed to potentially requiring multiple messages to learn about individual vehicles.

Ideal parameterization for VCAST: From all the above experimental evaluations, we note that an ideal parameterization for VCAST is to set communication range to a small value and increase the source broadcast rate. By doing so, information at smaller distances can be obtained at high rates with low contention due to the small communication range. At the same time, information can be transmitted to larger distances with progressively increasing staleness. For example, from Fig. 5.2 we notice that with $p=20 \mathrm{~Hz}$ and $r_{h}=200 \mathrm{~m}$, information about vehicles within $200 \mathrm{~m}$ can be obtained with a staleness of approximately $50 \mathrm{~ms}$ and information about vehicles within $700 \mathrm{~m}$ can be obtained with a staleness of approximately 100ms. Moreover, by limiting the propagation of individual vehicular information up to distances of about 500-1000m (for utilization by safety applications) and only propagating aggregate information beyond that, we observe that information from several miles ahead can also be obtained within a few seconds without the need for any communication infrastructure. As pointed out in Fig. 5.10(b), with a source transmission rate of $20 \mathrm{~Hz}$ and a broadcast range of $200 \mathrm{~m}$, aggregate traffic information from $5 \mathrm{Km}$ away can be communicated in less than 5 seconds. 


\section{Chapter 6}

\section{Conclusions}

Using the VCAST algorithm we are able to obtain the individual vehicle location and aggregate traffic information over multiple hops using wireless vehicular networks without the use of any expensive road-side infrastructure, or any special hardware or modifications to vehicular transmission standards. But in order to ensure scalability with the vehicular density and range of interest we adopt a method of traffic information propagation that decreases linearly with distance from source. This results in the reduction of communication rate per each node when compared to those protocols that do not use the distance-sensitivity in information forwarding. This ensures a lower channel contention and hence allows an higher source broadcast rate with better information quality at smaller distances while still able to propagate information to larger distances. We showed that even with these conditional forwarding rules put on the vehicular networks, we obtain the information with an error mentioned as staleness bounded by $O\left(d^{2}\right)$ where d is the communication hop distance from source of information. Even it is showed that the average communication cost does not depend on vehicular density and number of vehicles in the region.

Thus it can be inferred that VCAST can be used in improving the safety and ease of operation in vehicles by providing the information regarding collisions, approaching emergency vehicles and lane merging's. Along with safety, VCAST helps in dynamic routing and navigation techniques by providing the aggregate traffic information over an extended neighborhood. The performance of VCAST was validated using extensive ns-3 simulations of a DSRC communication model [19] under different network densities, source broadcast rates and communication ranges .

In future work, we would like to extend these simulations by integrating an urban traffic simulation model to study the effects of system in the urban environment where the errors are more likely to happen. Also we would like to integrate VCAST with control algorithms for vehicular safety and navigation that utilize information with distance-sensitive quality. We would like to design optimal control laws for vehicular acceleration under models of distancesensitive information availability, which ensure the safety of the integrated control- 
communication system. We would also like to integrate our vehicular traffic mapping service with navigation front-end for dynamic computation of alternate routes and evaluate the impact of distance sensitivity on the quality of navigation performance. 


\section{References}

[1] U.S. Department of Transportation. Achieving the Vision: From VII to IntelliDrive. Policy white paper, 2010.

[2] V. Kulathumani and Y. Fallah. Vcast: An infrastructure-less vehicular traffic information service with distance sensitive precision. In IEEE Vehicular Technology Conference (VTC), 2012.

[3] Y. Liu and F. Dion. Safety assessment of information delays on intelligent vehicle control system performance. In Proceedings of Transportation Research Board Conference, 2006.

[4] V. Kulathumani and A. Arora. Aspects of distance sensitive design of wireless sensor networks. In IEEE Workshop on Spatial Computing, 2008.

[5] V. Kulathumani, A. Arora, and S. Ramagiri. Pursuit control over wireless sensor networks using distance sensitivity properties. IEEE Transactions on Automatic Control, 56(10):2473-2478, 2011.

[6] J. B. Kenney. Dedicated Short-Range Communications (DSRC) Standards in the United States. Proceedings of the IEEE, 99(7):1162-1182, 2011.

[7] F. Li. Routing in vehicular ad hoc networks: A survey. IEEE Vehicular Technology Magazine, 2(2):12-22, 2007.

[8] K. C. Lee, U. Lee, and M. Gerla. Survey of routing protocols in vehicular ad hoc networks. In Advances in Vehicular Ad-Hoc Networks: Developments and Challenges, pages 149-170. IGI-Global, 2009.

[9] Y. Lin, Y. Chen, and S. Lee. Routing protocols in vehicular ad hoc networks: A survey and future perspectives. Journal of Information Science and Engineering, 26(2):913-932, 2010.

[10] A. Skordylis and N. Trigoni. Delay-bounded routing in vehicular ad-hoc networks. In ACM International Symposium on Mobile Ad hoc Networking and Computing, 2008.

[11] G. P. Joshi, M. Sichitiu, and M. Kihl. Distributed robust geocast multicast routing for inter-vehicle communication. In WEIRD Workshop on WiMax, Wireless and Mobility, 2007.

[12] H. Lu and C. Poellabauer. Balancing broadcast reliability and transmission range in VANETs. In IEEE Vehicular Networking Conference (VNC), 2010.

[13] L. Cheng and R. Shakya. VANET adaptive power control from realistic propagation and traffic modeling. In IEEE conference on Radio and wireless symposium, 2010.

[14] L. Yang, J. Guo, and Y. Wu. Channel Adaptive One Hop Broadcasting for VANETs. In IEEE Conference on Intelligent Transportation Systems, 2008.

[15] T. Osafune, L. Lin, and M. Lenardi. Multi-hop vehicular broadcast (MHVB). In International Conference on IntelligentTransportation Systems telecommunications (ITST), 2006.

[16] J. Fukuyama. A probabilistic protocol for multi-hop routing in VANETs. In IEEE International Conference on Communications Workshop, 2009.

[17] S. Ni, Y. Tseng, Y. Chen, and J. Sheu. The broadcast storm problem in a mobile ad hoc network. In ACM/IEEE international conference on Mobile computing and networking, 1999.

[18] B. Williams and T. Camp. Comparison of Broadcasting Techniques for Mobile Ad Hoc Networks. In ACM International Symposium on Mobile Ad Hoc Networking and Computing (MobiHoc), 2002.

[19] L. Cheng, B. Henty, D. Stancil, F. Bai, and P. Mudalige. Mobile Vehicle-to-Vehicle Narrow-Band Channel Measurement and Characterization of the $5.9 \mathrm{GHz}$ Dedicated Short Range Communication Frequency Band. IEEE Journal on Selected Areas in Communication (JSAC), 25(8):1501-1516, 2007

[20] E. W. Dijkstra. Self-stabilizing systems in spite of distributed control. Communications of the ACM, 17(11), 1974.

[21] V. Kulathumani and A. Arora. Distance sensitive snapshots in wireless sensor networks. In Principles of Distributed Systems (OPODIS), volume 4878, pages 143-158, 2007.

[22] M. Torrent-Moreno, J. Mittag, P. Santi, and H. Hartenstein. Vehicle-to-vehicle communication: Fair transmit power control for safety-critical information. IEEE Transactions on Vehicular Technology, 58(7):3684 -3703, 2009.

[23] http://www.nsnam.org/docs/tutorial/html/resources.html, NS-3 Resources, Accessed on May 25th 2012. 